\title{
Are two gluons the QCD Pomeron?
}

\author{
O. J. P. Éboli \\ Instituto de Física Teórica, Universidade Estadual Paulista, Rua Pamplona 145, 01405-900, São Paulo-SP, Brazil
}

E. M. Gregores and F. Halzen

Department of Physics, University of Wisconsin, Madison, Wisconsin 53706

(Received 6 May 1998; published 13 October 1998)

\begin{abstract}
Collider experiments have recently measured the production cross section of hard jets separated by a rapidity gap as a function of transverse momentum and gap size. We show that these measurements reveal the relative frequencies for the production of rapidity gaps in quark-quark, quark-gluon and gluon-gluon interactions. The results are at variance with the idea that the exchange of two gluons is a first order approximation for the mechanism producing colorless states, i.e. the hard QCD Pomeron. They do qualitatively support the "soft color" or "color evaporation" scheme developed in the context of bound-state heavy quark production.

[S0556-2821(98)09821-X]
\end{abstract}

PACS number(s): 12.38.Aw, 13.85.-t, 13.90.+i

\section{INTRODUCTION}

Although we do understand strong interactions in the context of QCD, we can at best speculate on how to calculate elastic scattering. This is just one example of an interaction mediated by the exchange of the "Pomeron," a state which carries no net color. The routine speculation has been that it is, to a first approximation, a state of two colored gluons combined into a color singlet. Understanding the Pomeron has been challenging because its dynamics is revealed in processes which are not subject to perturbative computation, e.g. elastic scattering. The hope has been that it may be instructive to study Pomeron dynamics in hard processes that are, at least partially, understood in terms of perturbative QCD: the hard Pomeron. Examples include processes involving colorless pairs of heavy quarks, e.g. $\psi$ 's, or colorless states of light quarks produced in association with a pair of high transverse momentum jets: rapidity gaps. In this paper we will show how a treatment of colorless states in QCD, suggested by the phenomenology of heavy quark bound states, supports a "soft color" model of the Pomeron which is at variance with the idea that it is a structure built on a frame composed of two gluons. We will show that recent measurements [1-3] of the relative frequency for the production of rapidity gaps in quark-quark, quark-gluon and gluongluon interactions provides qualitative, yet convincing, confirmation of the soft color concept.

The reason why some data on the production of $\psi$ and $Y$ states radically disagree with color singlet model predictions, occasionally by well over one order of magnitude, is that this method for performing the perturbative calculation of the cross section is simply wrong [4]. The key mistake is to require that the heavy quark pair forms a color singlet at short distances, given that there is an essentially infinite time for soft gluons to readjust the color of the $c \bar{c}$ pair before it appears as an asymptotic $\psi$ or, alternatively, $D \bar{D}$ state. We suspect that the same mistake is made in the description of rapidity gaps, i.e. the production of a color-neutral quarkantiquark pair, in terms of the exchange of a color neutral gluon pair. The $\psi$ is, after all, a color neutral $c \bar{c}$ pair and we have shown [4] in quantitative detail that it is indeed produced by exactly the same dynamics as $D \bar{D}$ pairs; its color happens to be bleached by soft final-state interactions. This approach to color is also suggestive of the unorthodox prescription for the production of rapidity gaps in deep inelastic scattering, proposed by Buchmüller and Hebecker [5].

In this paper we emphasize that the recent measurements on the formation of rapidity gaps between a pair of high transverse momentum jets shed new light on the problem of how to treat color in semi-hard interactions. When applied to the formation of gaps between a pair of high transverse momentum jets in hadron collisions, the "soft color" or "color evaporation" approach suggests a formation rate of gaps in gluon-gluon subprocesses which is similar, or smaller, than in quark-quark induced events. Consequently, formation of gaps should increase with increased transverse momentum, or reduced collision energy of the jets. This prediction happens to be antithetical to the one obtained in two-gluon exchange Pomeron models. We show that the data resolve the issue in favor of the "soft color" computational scheme and questions the relevance of approximating the exchange of a color-singlet, hard Pomeron as a pair of gluons. We also exhibit the predictions of the "soft color" model for the production of rapidity gaps at the LHC energy.

\section{ONIUM CALCULATIONS WITH SOFT COLOR: A BRIEF REMINDER}

The once conventional treatment of color in perturbative QCD calculations, i.e., the color singlet model, has run into serious problems in describing the data on the production of charmonium and upsilon states [6]. Specific proposals to solve the onium problem agree on the basic solution: onium production is a two-step process where a heavy quark pair is produced first. At this initial stage all perturbative diagrams are included, whether the $c \bar{c}$ pair forms a color singlet state or not. This is a departure from the textbook approach where only diagrams with the charm pair in a color singlet state are 


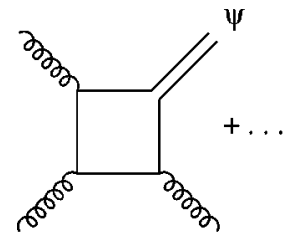

(a)

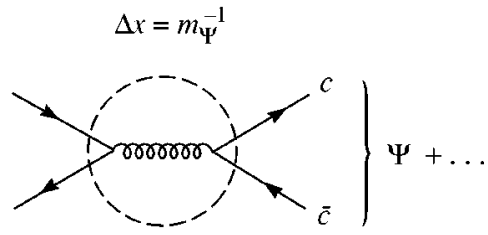

(b)
FIG. 1. Quarkonium production: (a) color singlet model; (b) color evaporation model.

selected. In the Bodwin-Braaten-Lepage (BBL) formalism [7] the subsequent evolution of the pair into a colorless bound state is described by an expansion in powers of the relative velocity of the heavy quarks in the onium system. A different approach, the color evaporation or soft color method, represents an even more radical departure from the way color states are conventionally treated in perturbation theory. Color is, in fact, "ignored." Rather than explicitly imposing that the system is in a given color state in the short-distance perturbative diagrams, the appearance of color singlet asymptotic states depends solely on the outcome of large-distance fluctuations of quarks and gluons. In other words, color is controlled by non-perturbative interactions.

In Fig. 1 we show typical diagrams for the production of $\psi$-particles representing the competing treatments of the color quantum number. In the diagram of Fig. 1(a), the color singlet approach, the $\psi$ is produced in gluon-gluon interactions in association with a final state gluon, which is required by color conservation. This diagram is related by crossing to the hadronic decay $\psi \rightarrow 3$ gluons. In the color evaporation approach, the color singlet property of the $\psi$ is ignored at the perturbative stage of the calculation. The $\psi$ can, for instance, be produced to leading order by $q \bar{q}$-annihilation into $c \bar{c}$, which is the color equivalent of the Drell-Yan process, as shown in Fig. 1(b). This diagram is calculated perturbatively, with dynamics dictated by short-distance interactions of range $\Delta x \simeq m_{\psi}^{-1}$. It does indeed not seem logical to enforce the color singlet property of the $\psi$ at short distances, given that there is an essentially infinite time for soft gluons to readjust the color of the $c \bar{c}$ pair before it appears as an asymptotic $\psi$ or, alternatively, $D \bar{D}$ state. Alternatively, it is indeed hard to imagine that a color singlet state formed at a range $m_{\psi}^{-1}$, automatically survives to form a $\psi$. This formalism represents the original [8-11] and, as we have shown [12], phenomenologically successful method by which perturbative QCD calculations should be performed.

The evidence is compelling that Nature operates according to the color evaporation scheme. The formalism predicts that, up to color and normalization factors, the energy, $x_{F}$ and $p_{T}$ dependences of the cross section, are identical for the production of onium states and $D \bar{D}$ pairs. This is indeed the case $[12,13]$. Another striking feature is that the production of charmonium is dominated by the conversion of a colored gluon into a $\psi$, as in Fig. 1(b). In the color singlet model, where the color singlet property of the $\psi$ is enforced at the perturbative level, three gluons (or two gluons and a photon) are required to produce a $\psi$. However, $\psi$ 's are not produced only by gluon-gluon interactions. As a consequence, color

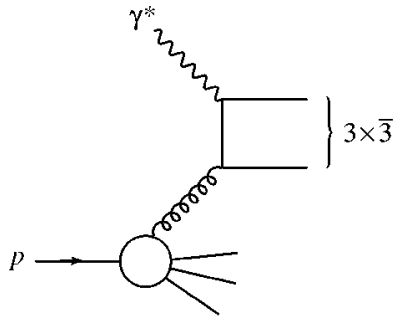

(a)

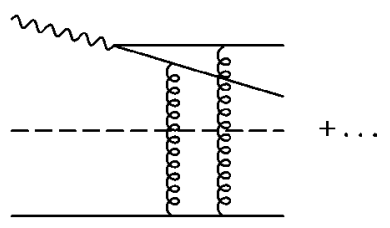

(b)
FIG. 2. Rapidity gaps in deep inelastic scattering: (a) due to soft colors effects; (b) due to Pomeron exchange.

evaporation predicts an enhanced $\psi$ cross section for antiproton beams, while the color singlet model predicts roughly equal cross sections for proton and antiproton beams. The prediction of an enhanced $\bar{p}$ yield is obviously correct: antiproton production of $\psi$ 's exceeds that by protons by a factor of 5 close to threshold. This fact has been known for some time [9-11]. We should note that for sufficiently high energies, gluon initial states will eventually dominate because they represent the bulk of soft partons.

Quantitative tests of color evaporation are made possible by the fact that all $\psi$-production data, i.e. photoproduction, hadroproduction, $Z$ decay, etc., are described in terms of a single parameter: the parameter determining the frequency by which a charm pair turns into a $\psi$ via the final state color fluctuations. Once this parameter has been empirically determined for one initial state, the cross section is predicted without free parameters for any other. We have demonstrated [13] the quantitative precision of the color evaporation scheme by showing how it accommodates all measurements, including the high energy Tevatron and HERA data [14], which have represented a considerable challenge for other computational schemes. Its parameter-free prediction of the rate for Z-boson decay into ' 's is an order of magnitude larger than the color singlet model and consistent with data [15].

In summary, the soft color approach gives a complete picture of charmonium production in hadron-hadron, $\gamma$ hadron, and $Z$ decays. The phenomenological success of the soft color scheme is impressive and extends to applications to other charmonium and upsilon states $[12,16]$.

\section{RAPIDITY GAPS AS COLORLESS STATES OF (LIGHT) QUARKS AND GLUONS}

We now turn to the implications of the soft color scheme for the dynamics underlying the production of rapidity gaps, which refer to regions in phase space where no hadrons appear as a result of the production of a color neutral partonic system. The connection to charmonium physics is obvious: the $\psi$ is a color-neutral $c \bar{c}$ pair. The important lesson from heavy quark phenomenology is that perturbative color octet states fully contribute to the asymptotic production of color singlet states, such as $\psi$ 's. We suspect that this is also true for the production of a rapidity gap which represents nothing but the creation of a color singlet quark-antiquark pair, as shown in Fig. 2(a) for electroproduction. The diagram repre- 


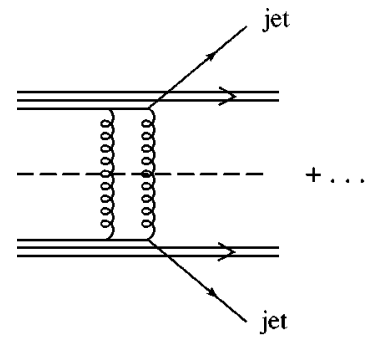

(a)

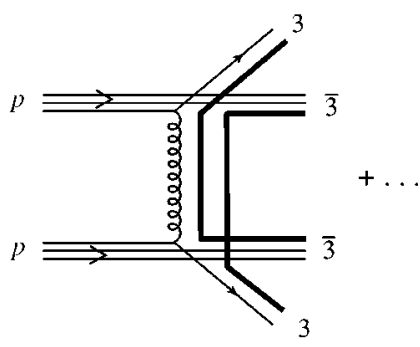

(b)
FIG. 3. Rapidity gap between jets: (a) due to Pomeron exchange; (b) due to soft color interactions.

sents the production of final state hadrons which are ordered in rapidity: From top to bottom we find the fragments of the intermediate partonic quark-antiquark state and those of the target. Buchmüller and Hebecker proposed that the origin of a rapidity gap corresponds to the absence of a color string between photon and proton remnants, i.e. the $\mathbf{3} \times \overline{\mathbf{3}}(=\mathbf{1}$ +8 ) intermediate quark-antiquark state is in a color singlet state. Because color is the source of hadrons, only the color octet states yield hadronic asymptotic states. The reasonable guess that

$$
F_{2}^{(g a p)}=\frac{1}{1+8} F_{2}
$$

follows from this argument.

Although this is only a guess, it embodies the essential physics: events with and without gaps are described by the same short-distance dynamics. Essentially non-perturbative final-state interactions dictate the appearance of gaps whose frequency may, possibly, be determined by simple counting. The treatment of color is the same as in the case of heavy quark production and leads to similar predictions: the same perturbative mechanisms, i.e. gluon exchange, dictates the dynamics of color-singlet gap $(\psi)$ and regular deep inelastic (open charm) events.

Our proposal for the (soft) nature of color challenges the orthodox mechanism for producing rapidity gaps sketched in Fig. 2(b), where the $t$-channel exchange of a pair of gluons in a color singlet state is the origin of the gap. The color string that connects photon and proton remnants in diagrams such as the one in Fig. 2(a) is absent and no hadrons are produced in the rapidity region separating them. The same mechanism predicts rapidity gaps between a pair of jets produced in hadronic collisions; see Fig. 3(a). These have been observed and occur with a frequency of order of one percent [17-19].

The arguments developed in this paper question the hard Pomeron approach: it is as meaningless to enforce the color singlet nature of the gluon pair as it is to require that the $c \bar{c}$ pair producing a $\psi$ is colorless at the perturbative level. Following our color scheme the gaps originate from a mere final state color bleaching phenomenon à la Buchmüller and Hebecker. This can be visualized using the diagram shown in Fig. 3(b). At short distances it represents a conventional perturbative diagram for the production of a pair of jets. There- fore, the same short distance dynamics governs events with and without rapidity gaps, as was the case for electroproduction. This is consistent with all experimental information.

Producing a quantitative model for the gap rate may be premature at this point. There are at least two consistent interpretations of the present data. The first is based on the string picture for the formation of the final state hadrons shown in Fig. 3(b). Color in the final state is bleached by strings connecting the $\mathbf{3}$ jet at the top with the $\overline{\mathbf{3}}$ spectator di-quark at the bottom and vice versa, resulting in color singlet states at the top and bottom. The probability to form a gap can be counted à la Buchmüller and Hebecker to be $1 /(1+8)^{2}$ because it requires the formation of singlets in two strings. The data [17-19] is consistent with this simple picture which basically predicts that the gap fraction between $p \bar{p}$ jets is the square of that between virtual photon and proton in deep inelastic scattering. One could, alternatively, argue that, once color has been bleached between one $3-\overline{\mathbf{3}}$ pair, overall color conservation will guarantee the color singlet value of the other pair. This leads to a gap formation rate which is similar in lepto- and hadroproduction, and can be reconciled with the data by introducing a survival probability. The survival probability accounts for the fact that gaps can be filled by the underlying event, e.g. mini-jet production [20], or by higher order processes. The survival probability of the gap is expected to be smaller for hadroproduction than electroproduction, thus accommodating the data.

Discussions of rapidity gap physics have routinely ignored that, besides quark-quark, gluon-gluon and gluonquark subprocesses contribute to jet production in hadron collisions. In the gluon-gluon color flow diagram corresponding to Fig. 3(b) top and bottom protons each split into a color octet gluon and a three-quark remnant in a color octet state. There are now $(8 \times 8)^{2}$ color final states. Despite the fact that we can at best guess the non-perturbative dynamics, it is clear that the soft color formalism predicts a gap rate which is smaller in gluon-gluon interactions. This is in contrast with the two-gluon exchange diagram of Fig. 3(a) which predicts a gap-rate enhanced by a factor $\left(\frac{9}{4}\right)^{2}$ in gluongluon subprocesses [21]. The process is clearly enhanced when replacing the interacting quarks by gluons, because of the larger gluon-gluon color coupling. The contrasting predictions can be easily tested by enhancing the relative importance of quark-quark subprocesses in the experimental sample. This can be achieved by increasing the $p_{T}$ of the jets at fixed energy, or by decreasing the collision energy of the hadrons at fixed $p_{T}$. In either case, we anticipate an increased rate for the production of gaps in the soft color scheme, a prediction opposite to that of the two-gluon exchange model. We next confront the contrasting predictions with recent data [1].

Introducing the quantities $F_{Q Q}, F_{Q G}$, and $F_{G G}$ which represent the frequencies for producing rapidity gaps between a pair of high- $p_{T}$ jets in hadronic quark-quark, quarkgluon, and gluon-gluon collisions, we can write the observed gap fraction as 


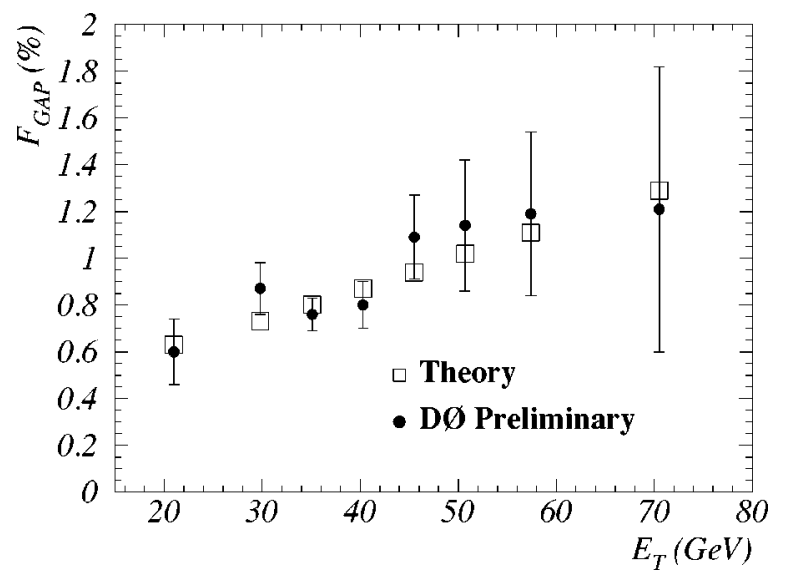

FIG. 4. Theoretical fit to the D0 preliminary data [1]. Notice that each point shown has a different $E_{T}$ bin, with the data being plotted at the mean value of $E_{T}$ for each bin, which is given in Table I.

$$
\begin{aligned}
F_{g a p}\left(E_{T}\right)= & \frac{1}{d \sigma / d E_{T}} \\
& \times\left(F_{Q Q} \frac{d \sigma_{Q Q}}{d E_{T}}+F_{Q G} \frac{d \sigma_{Q G}}{d E_{T}}+F_{G G} \frac{d \sigma_{G G}}{d E_{T}}\right),
\end{aligned}
$$

where

$$
d \sigma=d \sigma_{Q Q}+d \sigma_{Q G}+d \sigma_{G G}
$$

represents the decomposition of the cross section for producing large- $E_{T}$ jets into quark-quark, quark-gluon and gluongluon subprocesses. Predictions can be summarized in terms of the gap fractions $F_{i j}$. For the two-gluon hard Pomeron model [21],

$$
F_{Q Q}: F_{Q G}: F_{G G}=1: \frac{9}{4}:\left(\frac{9}{4}\right)^{2}
$$

In the soft color calculational scheme the $F_{i j}$ are independent of the center-of-mass energy and $E_{T}$, satisfying

$$
F_{Q Q}: F_{Q G}: F_{G G}=a: b: c,
$$

with $c<b<a$, in contrast with Eq. (4). Reasonable guesses fall in the range

$$
\begin{array}{r}
(1 / 9)^{2}<a<1 / 9, \\
a c<b<\sqrt{a c}, \\
(1 / 64)^{2}<c<1 / 64 .
\end{array}
$$

In order to determine the gap fractions $F_{i j}$, we computed the transverse cross sections $d \sigma_{i j} / d E_{T}$ in lowest order perturbative QCD, and subsequently fitted the preliminary D0 data [1] shown in Fig. 4. We integrated over the $E_{T}$ bins

\begin{tabular}{|c|c|c|c|c|}
\hline $\bar{E}_{T}(\mathrm{GeV})$ & $E_{T}$ bin $(\mathrm{GeV})$ & $\int \frac{d \sigma_{Q Q}}{d E_{T}}$ & $\begin{array}{c}\int \frac{d \sigma_{Q G}}{d E_{T}} \\
(\mathrm{nb})\end{array}$ & $\int \frac{d \sigma_{G G}}{d E_{T}}$ \\
\hline 21.0 & $15-25$ & 27.8 & 80.5 & 59.4 \\
\hline 29.8 & $25-30$ & 2.02 & 4.09 & 2.10 \\
\hline 35.1 & $30-35$ & 0.79 & 1.34 & 0.59 \\
\hline 40.3 & $35-40$ & 0.34 & 0.50 & 0.19 \\
\hline 45.5 & $40-45$ & 0.15 & 0.19 & 0.06 \\
\hline 50.7 & $45-50$ & 0.07 & 0.08 & 0.02 \\
\hline 57.4 & $50-60$ & 0.06 & 0.05 & 0.01 \\
\hline 70.5 & $>60$ & 0.03 & 0.02 & 0.004 \\
\hline
\end{tabular}
given in Table I, imposing that both jets have $|\eta|>1.9$. The 90\% C.L. bounds on the $F_{i j}$ are

$$
F_{Q Q}=0.022_{-0.010}^{+0.008}
$$

TABLE I. $E_{T}$ bins used in Fig. 4 and its respective mean value $\bar{E}_{T}$. For comparison, we also provide the cross section integrated over the bins for the quark-quark, quark-gluon, and gluon-gluon subprocesses.

$$
\begin{gathered}
F_{Q G}=0.00016_{-0.000}^{+0.011}, \\
F_{G G}=0.0072_{-0.0069}^{+0.0084} .
\end{gathered}
$$

We also show in Fig. 4 the result of our fit.

The D0 Collaboration has also measured the dependence of the fraction of events with rapidity gaps as a function of the rapidity separation of the leading jets for $E_{T}>30 \mathrm{GeV}$ and $|\eta|>1.7$ [22]. Our predictions are successfully confronted with their preliminary data [1] in Fig. 5.

The Collider Detector at Fermilab (CDF) measurements of the dependence of the fraction of gap events on transverse energy and on the separation in rapidity of the two jets are consistent with our interpretation of the D0 data. In Fig. 6 we present a comparison of the CDF results for both $\sqrt{s}=630$ and $1800 \mathrm{GeV}$, with the rapidity gap fraction predicted by our model. We here use the parameters extracted from the preliminary D0 data at $1800 \mathrm{GeV}$. We show the different contributions from quark-quark, quark-gluon, and gluongluon interactions to the transverse energy and rapidity size distributions. We also exhibit in Fig. 7 the dependence of the gap fraction on the fraction $x$ of the hadron momentum car-

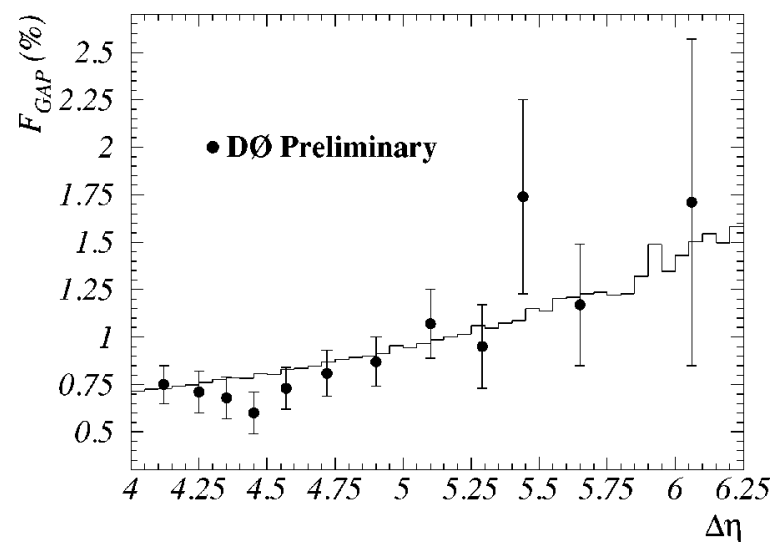

FIG. 5. Comparison with the preliminary D0 data [1] of our model prediction for the rapidity gap fraction as function of the size of the gap. 

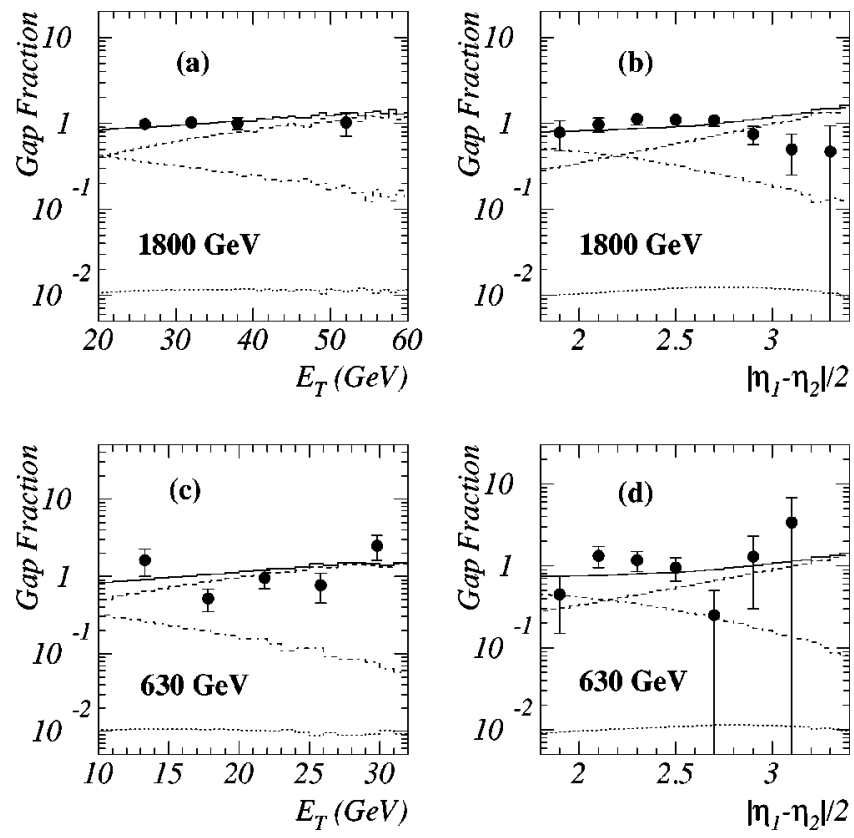

FIG. 6. Comparison with the CDF data [2] for $\sqrt{s}$ $=1800 \mathrm{GeV}$, and the preliminary CDF data [3] for $\sqrt{s}$ $=630 \mathrm{GeV}$. We show the different contributions of the quark-quark (dashed), quark-gluon (dotted) and gluon-gluon (dot-dashed) initial states, while the solid line stands for the sum of these contributions. The overall normalization has been appropriately scaled to correspond to the average fraction of rapidity gap events observed by CDF.

ried by the interacting parton. Like the data, our results have been normalized to unity, on average.

The analysis confirms that rapidity gaps are mostly formed in quark-quark collisions with the value of $F_{Q Q}$ ex-
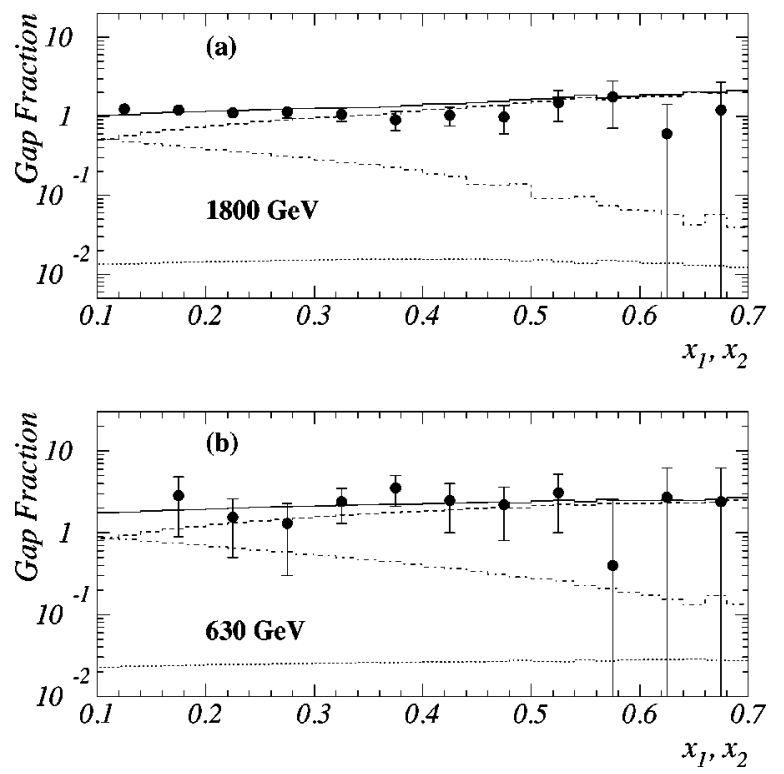

FIG. 7. Comparison with the preliminary CDF data [3] at $\sqrt{s}$ $=630$ and $1800 \mathrm{GeV}$ for the fraction of the rapidity gap events as a function of the fraction of the hadron momentum carried by the interacting parton. The conventions are the ones used in Fig. 6.

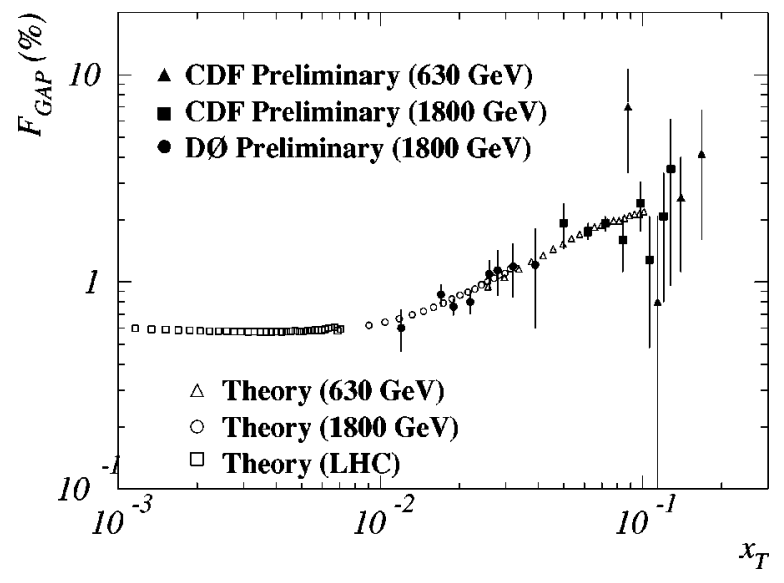

FIG. 8. Rapidity gap fraction as a function of the adimensional variable $x_{T}=E_{T} / \sqrt{s}$. For comparison we exhibit the preliminary D0 [1] and CDF data [3].

ceeding those of $F_{G G}$ and $F_{G Q}$. The $90 \%$ C.L. upper limits on $F_{Q G}$ and $F_{G G}$ are 0.011 and 0.016 , respectively.

Clearly, the data is consistent with the soft color model for the formation of rapidity gaps and does not support the two-gluon exchange approximation which predicts that processes involving gluons should exhibit larger rapidity gap frequencies. The data can be interpreted in terms of the simple color counting previously introduced, which predicts $F_{Q Q} \simeq(0.2-0.4) / 9 \simeq 2-4 \times 10^{-2}$ and $F_{G G} \simeq(0.2-0.4) / 64 \simeq 3-6 \times 10^{-3}$ for a survival probability [23] of $0.2-0.4$. This is certainly compatible with our results given the uncertain systematics of our procedure.

Our formalism has non-trivial dynamics built in. It assumes that the gap fractions $F_{i j}$ are independent of any kinematic variables. Therefore, the observed fraction of rapidity gaps depends on kinematical variables only through the relative contributions of the three subprocess cross sections $\sigma_{Q Q}, \sigma_{Q G}$, and $\sigma_{G G}$. In this case, the gap frequencies are only a function of the scaled variable $x_{T}=E_{T} / \sqrt{s}$, with the gap frequencies being described by a universal curve at all energies, as can be see in Fig. 8.

At the CERN Large Hadron Collider (LHC) energy, the fraction of events exhibiting rapidity gaps associated with moderate $E_{T}$ jets $(<80 \mathrm{GeV})$ is quite small $(\sim 0.6 \%)$ due to the large gluon-gluon luminosity. Notwithstanding, we anticipate $2 \times 10^{5}$ spectacular events for $E_{T}>200 \mathrm{GeV}$ and an integrated luminosity of $100 \mathrm{fb}^{-1}$ because the gap frequency increases to $\sim 0.8 \%$ in this $E_{T}$ range. On a more practical note, overlapping events may make such observations challenging.

The soft color mechanism can also give rise to rapidity gaps with a different ordering in rapidity: near the beam are the remnants of an initial hadron, followed by a rapidity gap and two hard jets which are separated from the other hadron debris by yet another rapidity gap. These events represent diffractive gaps. In the context of the color evaporation model, quark-quark and quark-gluon collisions cannot initiate such events because of the impossibility to neutralize the color of the $\mathbf{3}(\overline{\mathbf{3}})$ remnants with the exchange of soft gluons. Therefore, such events only originate in gluon-gluon 
collisions, and their fraction should decrease with increasing $E_{T}$, or decreasing center-of-mass energy.

We close with some cautionary comments. Do Figs. 2 and 3 suggest that we have formulated alternative $s$ - and $t$-channel pictures to view the same physics? Although they seem at first radically different, this may not be the case. Computation of the exchange of a pair of colorless gluons in the $t$ channel is not straightforward and embodies all the unsolved mysteries of constructing the "Pomeron" in QCD. In a class of models where the Pomeron is constructed out of gluons with a dynamically generated mass $[20,24]$, the diagram of Fig. 3(a) is, not surprisingly, dominated by the configuration where one gluon is hard and the other soft. The diagram is identical to the standard perturbative diagram except for the presence of a soft, long-wavelength gluon whose only role is to bleach color. Its dynamical role is minimal, events with gaps are not really different from events without, suggesting dynamics similar to color evaporation. Some have argued that in this class of models the hard Pomeron is no more than an order $\alpha_{s}^{2}$ correction, a view which can be defended on more solid theoretical ground [25]. Others have, however, challenged the theoretical soundness of this line of thinking $[23,26]$. Also note that our discussion is at best indirectly relevant to completely non-perturbative phenomena such as elastic scattering. There is no short distance limit defined by a large scale - the Pomeron exists.

\section{ACKNOWLEDGMENTS}

We would like to thank J. Amundson for collaborations and J. Bjorken, M. Drees, G. Ingelman, A. White, D Zeppenfeld, S. Fleming, B. May, J. Perkins, T. Taylor, and A. Brandt for their insight. This research was supported in part by the University of Wisconsin Research Committee with funds granted by the Wisconsin Alumni Research Foundation, by the U.S. Department of Energy under grant DEFG02-95ER40896, by Conselho Nacional de Desenvolvimento Científico e Tecnológico (CNPq), and by Fundação de Amparo à Pesquisa do Estado de São Paulo (FAPESP).
[1] D0 Collaboration, J. Perkins, in Proceedings of the 5th International Workshop on Deep Inelastic Scattering and QCD, Chicago, Illinois, 1997; D0 Collaboration, A. Abbott et al., talk presented at the 18th International Conference on Lepton Photon Interactions, Hamburg, 1997, FERMILAB-CONF-97/ 250-E.

[2] CDF Collaboration, F. Abe et al., Phys. Rev. Lett. 80, 1156 (1998).

[3] CDF Collaboration, K. Goulianos, in Proceedings of the LAFEX International School on High Energy Physics (LISHEP-98), Rio de Janeiro, Brazil, 1998, FERMILABCONF-98/118-E.

[4] O. J. P. Éboli, E. M. Gregores, and F. Halzen, in Proceedings of the 26th International Symposium on Multiparticle Dynamics (ISMD 96), Faro, Portugal, 1996, edited by J. Dias de Deus, P. Sa', M. Pimenta, S. Ramas, and J. Seixas (World Scientific, Singapore, 1997), p. 401.

[5] W. Buchmüller, Phys. Lett. B 353, 335 (1995); W. Buchmüller and A. Hebecker, ibid. 355, 573 (1995).

[6] See, e.g., E. Braaten, S. Fleming, and T. C. Yuan, Annu. Rev. Nucl. Part. Sci. 46, 197 (1997), and references therein.

[7] G. T. Bodwin, E. Braaten, and G. Lepage, Phys. Rev. D 51, 1125 (1995).

[8] H. Fritzsch, Phys. Lett. 67B, 217 (1977).

[9] F. Halzen, Phys. Lett. 69B, 105 (1977).

[10] F. Halzen and S. Matsuda, Phys. Rev. D 17, 1344 (1978).

[11] M. Glück, J. Owens, and E. Reya, Phys. Rev. D 17, 2324 (1978).

[12] J. Amundson, O. J. P. Éboli, E. M. Gregores, and F. Halzen, Phys. Lett. B 372, 127 (1996).
[13] J. Amundson, O. J. P. Éboli, E. M. Gregores, and F. Halzen, Phys. Lett. B 390, 323 (1997).

[14] O. J. P. Éboli, E. M. Gregores, and F. Halzen, MADPH-981045, 1998, hep-ph/9802421.

[15] O. J. P. Éboli, E. M. Gregores, and F. Halzen, Phys. Lett. B 395, 113 (1997).

[16] R. Gavai et al., Int. J. Mod. Phys. A 10, 3043 (1995); R. Vogt and G. Schuler, Phys. Lett. B 387, 181 (1996).

[17] D0 Collaboration, S. Abachi et al., Phys. Rev. Lett. 72, 2332 (1994).

[18] CDF Collaboration, F. Abe et al., Phys. Rev. Lett. 74, 855 (1995).

[19] D0 Collaboration, S. Abachi et al., Phys. Rev. Lett. 76, 734 (1996).

[20] H. Chehime et al., Phys. Lett. B 286, 397 (1992).

[21] D. Zeppenfeld, in Proceedings of the VIII J. A. Swieca Summer School: Particles and Fields, Brazil, edited by S. F. Novaes and V. O. Rivelles (World Scientific, Singapore, 1996).

[22] T. Thomas, talk given at the 3rd Workshop on Small $\mathrm{x}$ and Diffractive Physics, Argonne, Sept. 26-29, 1996.

[23] J. D. Bjorken, Int. J. Mod. Phys. A 7, 4189 (1992); Phys. Rev. D 47, 101 (1993); SLAC-PUB-5823, 1992.

[24] F. Halzen, G. Krein, and A. A. Natale, Phys. Rev. D 47, 295 (1993); M. B. Gay Ducati, F. Halzen, and A. Natale, ibid. 48, 2324 (1993).

[25] J. R. Cudell, A. Donnaichie, and P. V. Landshoff, Nucl. Phys. B482, 241 (1996).

[26] A. White, talk given at the 3rd Workshop on Small $\mathrm{x}$ and Diffractive Physics [22]. 